\title{
Seletividade de Imazapic Às Soqueiras de Cana-de-Açúcar (Saccharum Spp.) ${ }^{1}$
}

\author{
Imazapic Selectivity to Sugarcane Ratoons (Saccharum spp.)
}

AZANIA, C.A.M. ${ }^{2}$, CASAGRANDE, A.A. ${ }^{3}$ e ROLIM, J.C. ${ }^{4}$

\begin{abstract}
RESUMO - Esta pesquisa objetivou a avaliar a seletividade de imazapic às soqueiras de cana-de-açúcar, cultivar RB 835089, comparado a imazapyr e tebuthiuron. O estudo foi conduzido no município de Araras-SP, durante o período de 1999/2000, em solo de classe textural franco-argilo-arenosa, devidamente corrigido e adubado. A instalação do experimento foi em blocos, com sete tratamentos casualizados e cinco repetições, a saber: imazapic $(73,5$; 98,0; e 122,5 $\left.\mathrm{g} \mathrm{ha}^{-1}\right)$, imazapyr (125,0 $\left.\mathrm{g} \mathrm{ha}^{-1}\right)$ e tebuthiuron (1.100,0 $\left.\mathrm{g} \mathrm{ha}^{-1}\right)$. Os herbicidas foram aplicados em pós-emergência inicial da cultura, além das testemunhas com e sem capinas. Foi possivel concluir que todos os produtos testados causaram leves sintomas de intoxicação inicial nas plantas de cana-de-açúcar, com total recuperação aos 100 dias após os tratamentos, sem prejuízo da produtividade e qualidade da matéria-prima.
\end{abstract}

Palavras-chave: tolerância, herbicidas.

ABSTRACT - This research aimed to evaluate imazapic selectivity to sugarcane ratoons, compared to imazapyr and tebuthiuron, applied in early post emergence at Araras-São Paulo 1999/2000. The study was conducted in a soil with $21 \%$ clay and $71 \%$ sand, properly corrected and fertilized. The experimental design was a randomized complete block with five replications of seven treatments: imazapic (73.5, 98.0 and $\left.122.5 \mathrm{~g} \mathrm{ha}^{-1}\right)$, imazapyr $\left(125.0 \mathrm{~g} \mathrm{ha}^{-1}\right)$ and tebuthiuron (1100.0 $\left.\mathrm{g} \mathrm{ha}^{-1}\right)$ and controls with and without weeds. All the herbicides caused initial toxicity to the sugarcane ratoons, until 100 days after treatments, without damage to productivity and raw material quality.

Key words: tolerance, herbicides.

\section{INTRODUÇÃO}

O imazapic é um herbicida registrado para as culturas do amendoim e da soja, proporcionando excelente controle de plantas daninhas com produtividade satisfatória, mesmo causando injúrias na fase inicial das culturas. Muitos autores, como Akanda et al. (1997), Grichar (1997a, b), Richard Jr. (1997) e outros, representam-no em suas pesquisas pelo código AC-263,222 e nome químico (+/-)-2-[4,5dihydro-4-methyl-4-(1-methylethyl)-5-oxo-1Himidazol-2-yl]-5-methyl-3 pyridinecarboxylic acid. Na cana-de-açúcar ele ainda tem uso limitado e pouco se conhece a respeito dos sintomas de intoxicação nas plantas da cultura, sendo necessárias mais pesquisas para demonstrar esses efeitos. Em outras culturas, como a soja, encontram-se trabalhos como o de Shaw \& Wixson (1991), que constataram injúrias de 8\% à cultura, 56 dias após tratamento (DAT), quando se aplicou, em pós-emergência, o imazapic (70 $\left.\mathrm{g} \mathrm{ha}^{-1}\right)$ combinado com imazaquim (140 $\mathrm{g} \mathrm{ha}^{-1}$ ).

O estádio da planta no momento da aplicação influi na seletividade do herbicida. Wixson \& Shaw (1991b) constataram que o imazapic aplicado no estádio mais inicial da cultura da

1 Recebido para publicação em 27/11/2000 e na forma revisada em 17/12/2001.

2 Mestrando em Produção Vegetal, FCAV/UNESP, 14870-000 Jaboticabal-SP; ${ }^{3}$ Prof. Dr. no Departamento de Produção Vegetal, FCAV/UNESP. ${ }^{4}$ Prof. Dr. no Departamento de Recursos Naturais, CCA/UFSCar, 13600-000 Araras-SP. 
soja foi mais tóxico. O uso de imazaquim (140 $\mathrm{g} \mathrm{ha}^{-1}$ ) em pré-emergência, seguido de imazapic (35 $\left.\mathrm{g} \mathrm{ha}^{-1}\right)$ em pós-emergência inicial, proporcionou injúrias de até $13 \%$, aos 56 DAT. Segundo Wixson \& Shaw (1992), a aplicação em pré-plantio de imazapic $\left(9,18\right.$ e $\left.35 \mathrm{~g} \mathrm{ha}^{-1}\right)$ também causou até $20 \%$ de injúria inicial em diferentes culturas. Além das doses e do modo de aplicação, Wixson \& Shaw (1991a) demonstraram que a adição de surfatante ou óleo concentrado colabora para o aumento da toxicidade na soja.

Para minimizar os efeitos fitotóxicos do produto, pode-se associá-lo ao cultivo, uma vez que, conforme Newsom \& Shaw (1994), doses menores de imazapic ( $\left.9 \mathrm{~g} \mathrm{ha}^{-1}\right)$, em pósemergência, seguidas de cultivo aos sete dias após o tratamento, proporcionaram menores injúrias. O uso de variedades tolerantes de soja também contribuiu para a diminuição dos percentuais de toxicidade, de acordo com Griffin et al. (1993).

Em cana-de-açúcar, Richard Jr. (1997) constatou que imazapyr ( $\left.1.120 \mathrm{~g} \mathrm{ha}^{-1}\right)$ não alterou a quantidade de açúcar teoricamente recuperável (ATR) e a sua produtividade. Richard Jr. (1998) também verificou que imazapyr (284 $\mathrm{g} \mathrm{ha}^{-1}$ ), aplicado por duas vezes, reduziu o estande, a altura e o ATR da cana-de-açúcar.

O tebuthiuron é um herbicida tradicionalmente utilizado em cana-de-açúcar, porém também apresenta problemas de toxicidade. Segundo Coleti \& Nunes (1982), existem diferenças entre variedades quanto à tolerância a esse herbicida. Esses autores constataram que as variedades IAC 48-65, IAC 52-150, NA 56-79, SP 70-1143 e SP 71-3149 são mais tolerantes que IAC 51-205, CB45-155, CP 5122 e SP 70-1078. Entretanto, no trabalho de Rolim \& Christoffoleti (1982) não foram verificadas alterações de produtividade e qualidade agrícola nas variedades NA 56-76, CB 41-78 e IAC 52-150 submetidas à aplicação de tebuthiuron $(800,1.200,1.600,1.800 \mathrm{e}$ $2.400 \mathrm{~g} \mathrm{ha}^{-1}$ ).

Diante da escassa literatura sobre os efeitos do imazapic sobre as plantas de cana-deaçúcar, objetivou-se avaliar neste trabalho a seletividade de imazapic para a cultura da cana-de-açúcar, soqueira de segundo corte do cultivar RB 835089, comparado a imazapyr e tebuthiuron.

\section{MATERIAL E MÉTODOS}

O experimento foi instalado no município de Araras-SP, no sítio São José, em solo com $21 \%$ de argila, $71 \%$ de areia, $8 \%$ de silte e $16 \mathrm{~g} \mathrm{dm}^{-3}$ de matéria orgânica. Os teores de macro e micronutrientes do solo da área experimental encontravam-se em condições satisfatórias, de acordo com a análise feita. A cultura estudada foi a cana-de-açúcar, soqueira de segundo corte do cultivar RB 835089. Os herbicidas e doses utilizados foram imazapic a 73,5; 98,0; e 122,5 $\mathrm{g} \mathrm{ha}^{-1}$, imazapyr a $125 \mathrm{~g} \mathrm{ha}^{-1}$ e tebuthiuron a $1.100 \mathrm{~g} \mathrm{ha}^{-1}$, com $0,25 \%$ de surfatante aplicado em pós-emergência inicial da cultura. O delineamento experimental foi o de blocos casualizados, com sete tratamentos e cinco repetições. As parcelas foram constituídas de cinco linhas de canade-açúcar com $10 \mathrm{~m}$ de comprimento, espaçadas de $1,5 \mathrm{~m}$, sendo consideradas úteis apenas as três linhas centrais, que constituíam uma área de $45 \mathrm{~m}^{2}$. A análise de variância pelo teste $\mathrm{F}$, de acordo com o delineamento em blocos casualizados, foi utilizada para avaliar o efeito dos tratamentos sobre as variáveis analisadas; posteriormente, na comparação das médias dos tratamentos, utilizou-se o teste de Tukey a 5\% de probabilidade.

A seletividade dos herbicidas às plantas de cana-de-açúcar foi avaliada visualmente aos $15,30,60$ e 100 DAT, nas três linhas centrais de cada parcela. Foram atribuídas notas de acordo com os sintomas de intoxicação na parte aérea das plantas de cana-de-açúcar, utilizando-se uma escala variando de 1 a 9 , sendo 1 para a toxicidade nula e 9 para a morte das plantas de cana-de-açúcar (EWRC, 1964). A altura (do solo até ao "dew-lap") de 10 plantas escolhidas ao acaso e o estande (contagem dos colmos) das plantas de cana-de-açúcar foram determinados nas três linhas centrais de cada parcela aos 90 e 180 DAT, sendo o estande também determinado no momento da colheita. Os valores de Brix (\%), Po (1\%), pureza (\%), fibra (\%) açúcares redutores (AR) e açúcar teórico recuperável (ATR) foram determinados pelas amostragens de 12 colmos na linha central de cada parcela, quatro dias antes da colheita da cana-de-açúcar. No momento da colheita foi determinado o peso $\left(\mathrm{t} \mathrm{ha}^{-1}\right)$ dos colmos das três linhas centrais de cada parcela. 


\section{RESULTADOS E DISCUSSÃO}

Observa-se, pela Figura 1, que os herbicidas apresentaram, no máximo, toxicidade de nota 3, correspondente a sintomas de intoxicação leves e aceitos na prática. Os principais sintomas visuais observados nas folhas das plantas de cana-de-açúcar foram manchas cloróticas, estrias e leve enrolamento em algumas folhas.

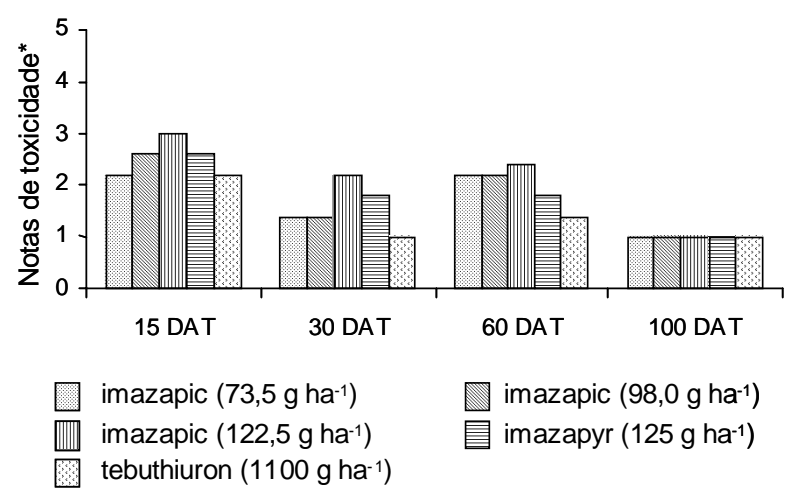

* Nota 1: ausência de toxicidade; nota 2: sintomas muito leves; nota 3: leves aceitos na prática.

Figura 1 - Média das notas visuais de toxicidade causadas pelos herbicidas nas plantas de cana-de-açúcar, segundo a escala da EWRC (1964), no período de 1999/2000, em Araras-SP.

Aos 15 DAT, todos os herbicidas testados causaram baixa intoxicação à cultura, e as plantas de cana-de-açúcar apresentaram sintomas de intoxicação muito leves (nota 2) e leves aceitos na prática (nota 3), segundo a escala EWRC. Nesta mesma avaliação, observou-se que, para imazapic, os sintomas na cultura aumentaram proporcionalmente à dose testada, atingindo nota 3 apenas com imazapic na maior dose. Os tratamentos com tebuthiuron e imazapic a 73,5 $\mathrm{g} \mathrm{ha}^{-1}$ causaram os menores sintomas de intoxicação à cultura, enquanto o tratamento com imazapyr apresentou os mesmos sintomas que aquele com imazapic na dose intermediária.

Aos 30 DAT, apenas imazapic a $122,5 \mathrm{~g} \mathrm{ha}^{-1}$ proporcionou sintomas de intoxicação muito leves (nota 2), enquanto para imazapyr, tebuthiuron e para as demais doses de imazapic os sintomas de intoxicação foram quase que totalmente ausentes, apresentando nota 1 . Esses dados demonstraram que as doses de imazapic utilizadas foram adequadas para as plantas de cana-de-açúcar, pois, segundo a pesquisa de Wixson \& Shaw (1992), o uso de imazapic a $35 \mathrm{~g} \mathrm{ha}^{-1}$ nas culturas de algodão e milho proporcionou sintomas de intoxicação mais elevados aos 28 DAT.

Aos 60 DAT, com exceção de imazapyr, todos os herbicidas voltaram a proporcionar sintomas de intoxicação mais elevados na cultura. A explicação para esse acontecimento se deve às condições climáticas atípicas registradas nos dois meses anteriores e nos três posteriores ao da instalação do experimento, com valores pluviométricos inferiores e com temperaturas superiores àquelas normalmente observadas na região, conforme demonstrado na Tabela 1. Desde a instalação do experimento, na segunda quinzena de setembro, até o final de dezembro, constatou-se total de precipitações de 223,80 mm, contra 568,70 mm nos anos antecessores, sendo essa diferença correspondente a $154,11 \%$ menor. O défice hídrico existente proporcionou o aparecimento de manchas e enrolamento de folhas nas plantas da cultura, e essas características influenciaram a avaliação visual dos sintomas de intoxicação nas plantas de cana-de-açúcar, dificultando a identificação dos sintomas de herbicidas.

Tabela 1 - Precipitações pluviais e temperaturas médias ocorridas nos últimos dez anos (1989-1999) e durante a condução do experimento, em Araras-SP

\begin{tabular}{|l|r|c|c|c|}
\hline \multirow{2}{*}{ Mês } & \multicolumn{2}{|c|}{$\begin{array}{c}\text { Precipitação } \\
(\mathrm{mm})\end{array}$} & \multicolumn{2}{c|}{$\begin{array}{r}\text { Temperatura média } \\
\left({ }^{\circ} \mathrm{C}\right)\end{array}$} \\
\cline { 2 - 5 } & Média* & Experimento & Média** & Experimento \\
\hline Julho & 24,5 & 0,0 & 16,7 & 21,2 \\
Agosto & 27,9 & 0,0 & 18,6 & 20,4 \\
Setembro & 59,4 & 108,$0 ; 11,6^{* * *}$ & 20,1 & 21,6 \\
Outubro & 133,3 & 45,6 & 20,8 & 21,4 \\
Novembro & 146,6 & 39,0 & 21,4 & 22,1 \\
Dezembro & 229,4 & 127,6 & 21,9 & 25,7 \\
\hline
\end{tabular}

Fonte: CCA/UFSCar.

* - média das precipitações nos últimos dez anos.

** - média das temperaturas nos últimos dez anos.

*** - chuvas ocorridas após a instalação do experimento realizado em 14/09/1999.

Planta Daninha, Viçosa-MG, v.19, n.3, p.345-350, 2001 
Mesmo com o défice hídrico, aos 60 DAT, observaram-se sintomas muitos leves de intoxicação nas plantas de cana-de-açúcar. Entretanto, com a volta da regularidade pluviométrica, aos 100 DAT, pôde-se constatar que a cultura não apresentou nenhum sintoma de intoxicação nos tratamentos envolvidos, recuperando-se totalmente.

Outra evidência de que o imazapic, nas três doses, não interferiu no desenvolvimento da cultura são os dados de altura, estande, produtividade e qualidade tecnológica observados nas Tabelas 2 e 3 , onde não foi verificada nenhuma diferença estatística entre as características analisadas. Portanto, pode-se observar que os três herbicidas apresentaram toxicidade inicial similar às plantas de canade-açúcar.

O cultivar de cana-de-açúcar utilizado demonstrou ser tolerante aos herbicidas e suas respectivas doses, uma vez que a cultura se recuperou totalmente aos 100 DAT, não sendo observado nenhum comprometimento da qualidade tecnológica e produção final.

Tabela 2 - Efeito da aplicação de imazapyr e tebuthiuron sobre o estande (número de colmos por metro linear) e a altura (cm) das plantas de cana-de-açúcar, no período de 1999/2000, em Araras-SP

\begin{tabular}{|l|c|c|c|c|c|}
\hline \multirow{2}{*}{ Tratamentos } & \multicolumn{3}{|c|}{ Colmos/m } & \multicolumn{2}{c|}{ Altura (cm) } \\
\cline { 2 - 6 } & 90 DAT & 180 DAT & Colheita & 90 DAT & 180 DAT \\
\hline Imazapic $\left(73,5 \mathrm{~g} \mathrm{ha}^{-1}\right)$ & $11,9 \mathrm{a}$ & $9,9 \mathrm{a}$ & $10,9 \mathrm{a}$ & $71,7 \mathrm{a}$ & $198,6 \mathrm{a}$ \\
Imazapic $\left(98 \mathrm{~g} \mathrm{ha}^{-1}\right)$ & $11,2 \mathrm{a}$ & $10,2 \mathrm{a}$ & $10,8 \mathrm{a}$ & $73,1 \mathrm{a}$ & $196,8 \mathrm{a}$ \\
Imazapic $\left(122,5 \mathrm{~g} \mathrm{ha}^{-1}\right)$ & $10,9 \mathrm{a}$ & $10,0 \mathrm{a}$ & $11,1 \mathrm{a}$ & $72,4 \mathrm{a}$ & $198,4 \mathrm{a}$ \\
Imazapyr $\left(125 \mathrm{~g} \mathrm{ha}^{-1}\right)$ & $11,4 \mathrm{a}$ & $10,2 \mathrm{a}$ & $11,0 \mathrm{a}$ & $69,8 \mathrm{a}$ & $190,4 \mathrm{a}$ \\
Tebuthiuron $\left(1.100 \mathrm{~g} \mathrm{ha}^{-1}\right)$ & $11,9 \mathrm{a}$ & $9,9 \mathrm{a}$ & $11,1 \mathrm{a}$ & $71,7 \mathrm{a}$ & $196,0 \mathrm{a}$ \\
Testemunha capinada & $11,1 \mathrm{a}$ & $9,6 \mathrm{a}$ & $10,8 \mathrm{a}$ & $69,5 \mathrm{a}$ & $202,0 \mathrm{a}$ \\
Testemunha sem capina & $11,0 \mathrm{a}$ & $9,9 \mathrm{a}$ & $10,9 \mathrm{a}$ & $71,7 \mathrm{a}$ & $198,6 \mathrm{a}$ \\
\hline F (tratamento) & $1,35^{\mathrm{ns}}$ & $1,24^{\mathrm{ns}}$ & $0,16^{\mathrm{ns}}$ & $0,40^{\mathrm{ns}}$ & $1,11^{\mathrm{ns}}$ \\
CV $(\%)$ & 6,69 & 4,22 & 5,12 & 6,49 & 3,84 \\
dms & 1,5 & 0,9 & 1,1 & 9,4 & 15,39 \\
\hline
\end{tabular}

dms: diferença mínima significativa; ns: não-significativo a 5\% de probabilidade; CV: coeficiente de variação; DAT: dias após tratamento.

Tabela 3 - Efeitos da aplicação de imazapyr e tebuthiuron sobre a produção e análise tecnológica da cana-de-açúcar, no período de 1999/2000, em Araras-SP

\begin{tabular}{|c|c|c|c|c|c|c|c|}
\hline \multirow{2}{*}{ Tratamentos } & \multirow{2}{*}{$\begin{array}{c}\text { Produção } \\
\left(\mathrm{t} \mathrm{ha}^{-1}\right)\end{array}$} & \multicolumn{5}{|c|}{$\%$} & \multirow{2}{*}{$\begin{array}{c}\text { ATR } \\
\left(\mathrm{kg} \mathrm{t}^{-1}\right)\end{array}$} \\
\hline & & BRIX & POL & FIBRA & PUREZA & AR & \\
\hline Imazapic $\left(73,5 \mathrm{~g} \mathrm{ha}^{-1}\right)$ & $108,73 \mathrm{a}$ & $22,18 \mathrm{a}$ & $20,42 \mathrm{a}$ & $12,86 \mathrm{a}$ & $92,06 \mathrm{a}$ & $0,28 \mathrm{a}$ & $159,85 \mathrm{a}$ \\
\hline Imazapic $\left(98,0 \mathrm{~g} \mathrm{ha}^{-1}\right)$ & $107,02 \mathrm{a}$ & $22,28 \mathrm{a}$ & $20,42 \mathrm{a}$ & $12,84 \mathrm{a}$ & $91,80 \mathrm{a}$ & $0,31 \mathrm{a}$ & $160,09 \mathrm{a}$ \\
\hline Imazapic $\left(122,5 \mathrm{~g} \mathrm{ha}^{-1}\right)$ & $104,98 \mathrm{a}$ & $22,02 \mathrm{a}$ & $20,19 \mathrm{a}$ & $12,95 \mathrm{a}$ & $91,68 \mathrm{a}$ & $0,32 \mathrm{a}$ & $158,08 \mathrm{a}$ \\
\hline Imazapyr $\left(125 \mathrm{~g} \mathrm{ha}^{-1}\right)$ & $104,76 \mathrm{a}$ & $22,16 \mathrm{a}$ & $20,32 \mathrm{a}$ & $13,89 \mathrm{a}$ & $91,70 \mathrm{a}$ & $0,32 \mathrm{a}$ & $156,54 \mathrm{a}$ \\
\hline Tebuthiuron $\left(1.100 \mathrm{~g} \mathrm{ha}^{-1}\right)$ & $111,35 \mathrm{a}$ & $21,76 \mathrm{a}$ & $20,07 \mathrm{a}$ & $13,49 \mathrm{a}$ & $92,24 \mathrm{a}$ & $0,26 \mathrm{a}$ & $155,34 \mathrm{a}$ \\
\hline Testemunha capinada & $104,78 \mathrm{a}$ & $21,92 \mathrm{a}$ & $20,25 \mathrm{a}$ & $12,83 \mathrm{a}$ & $92,38 \mathrm{a}$ & $0,25 \mathrm{a}$ & $158,39 \mathrm{a}$ \\
\hline Testemunha sem capina & $103,46 \mathrm{a}$ & $22,04 \mathrm{a}$ & $20,36 \mathrm{a}$ & $12,87 \mathrm{a}$ & $92,38 \mathrm{a}$ & $0,25 \mathrm{a}$ & $159,17 \mathrm{a}$ \\
\hline $\mathrm{F}$ (tratamento) & $1,36^{\mathrm{ns}}$ & $1,43^{\mathrm{ns}}$ & $0,74^{\mathrm{ns}}$ & $0,48^{\mathrm{ns}}$ & $1,89^{\mathrm{ns}}$ & $1,95^{\mathrm{ns}}$ & $1,17^{\mathrm{ns}}$ \\
\hline $\mathrm{CV}(\%)$ & 5,00 & 1,49 & 1,65 & 10,28 & 0,55 & 18,76 & 2,28 \\
\hline dms $(5 \%)$ & 10,81 & 0,67 & 0,68 & 2,74 & 1,03 & 0,11 & 7,31 \\
\hline
\end{tabular}

* Análises realizadas pela Associação dos Fornecedores de Cana de Piracicaba.

dms: diferença mínima significativa; ns: não-significativo a 5\% de probabilidade; CV: coeficiente de variação. 
Os resultados de produção e Pol\%cana encontrados nos tratamentos com tebuthiuron estão de acordo com os trabalhos de Rolim \& Christoffoleti (1982) e Graciano \& Ramalho (1982), que utilizaram doses mais elevadas que aquela aplicada no experimento e também não obtiveram nenhum comprometimento de produção e qualidade tecnológica. Resultados similares de ATR e produtividade em $\mathrm{t} \mathrm{ha}^{-1}$ observados com a aplicação de imazapyr (125 $\mathrm{g} \mathrm{ha}^{-1}$ ) também foram obtidos na pesquisa de Richard Jr. (1997), mesmo aplicando imazapyr na dose de $1.120 \mathrm{~g} \mathrm{ha}^{-1}$. Outrora, ocorreram reduções de altura, estande e ATR quando Richard Jr. (1998) aplicou imazapyr a $284 \mathrm{~g} \mathrm{ha}^{-1}$ por duas vezes.

A toxicidade que o herbicida imazapic, nas três doses testadas, proporcionou às plantas de cana-de-açúcar foi similar à observada no uso de imazapyr e tebuthiuron, que possuem um histórico de uso na cultura da cana-deaçúcar. Portanto, pode-se concluir que imazapic nas três doses testadas, imazapyr e tebuthiuron causam leves sintomas de intoxicação na fase inicial de desenvolvimento da cultura, com total recuperação aos 100 DAT, sem prejuízo à produtividade e qualidade da matéria-prima.

\section{AGRADECIMENTOS}

À CAPES, pela ajuda financeira; à Associação dos Fornecedores de Cana de Piracicaba, pela realização das análises tecnológicas; aos professores Júlio César Durigan e José C. Barbosa, pelas sugestões; e aos proprietários rurais Percival e Irineu Pastre, pela concessão da área do experimento, das máquinas e da mão-de-obra no momento da colheita.

\section{LITERATURA CITADA}

AKANDA, R.V.; MULLAHEY, J. J.; DOWLER, C.C.; SHILLING, D.G. Influence of postemergence herbicides on tropical soda apple (Solanum viarum) and bahiagrass (Paspalum notatum). Weed Technol., v.11, p. 656-661, 1997.

COLETI, J.T.; NUNES Jr., D. Avaliação inicial da fitotoxicidade de herbicidas sobre 16 cultivares de cana-de-açúcar (Saccharum spp.). Campinas. In: CONGRESSO BRASILEIRO DE HERBICIDAS E ERVAS DANINHAS (SBHED), 14; CONGRESO DE LA ASSOCIACION LATINO AMERICANA DE MALEZAS (ALAM), 6, 1982, Campinas. Resumos... Campinas: 1982. p.89
EUROPEAN WEED RESEARCH COUNCIL. Report of the 3rd and 4rd meetings of EWRC. Comittes of Methods in Weed Research. Weed Res. Oxford, v.4, p.88, 1964.

GRACIANO, P.C.; RAMALHO, J.F.G.P. Suscetibilidade ao cultivar CB 45-3 de canade-açúcar (Saccharum spp) ao herbicida tebuthiuron. Campinas. CONGRESSO BRASILEIRO DE HERBICIDAS E ERVAS DANINHAS (SBHED), 14; CONGRESO DE LA ASSOCIACION LATINO AMERICANA DE MALEZAS (ALAM), 6, 1982, Campinas. Resumos... Campinas: 1982. p.91.

GRICHAR, W.J. Influence of herbicides and timing of application on broadleaf control in peanut (Arachis hypogaea). Weed Technol., v.11, p. 708-713, 1997a.

GRICHAR, W. J. Control of palmer amaranth (Amaranthus palmeri) in peanut (Arachis hypogaea) with postemergence herbicides. Weed Technol., v.11, p.739-743, 1997b.

GRIFFIN, J.L.; REYNOLDS, D. B.; VIDRINE, P. R.; BRUFF, S. A. Soybean (Glycine max) tolerance and sicklepod (cassia obtusifolia) control with AC 263,222. Weed Technol., v.17, p.331-336, 1993.

NEWSOM, L.J.; SHAW, D.R. Influence of cultivation timing on weed control in soybean (Glycine max) with AC 263,222. Weed Technol., v.8, p. 760$765,1994$.

RICHARD Jr., E.P. Effects of fallow bermudagrass (Cynodon dactylon) control programs on newly planted sugarcane (Saccharum spp hybrids). Weed Technol., v.11, p. 677-682, 1997.

RICHARD Jr., EP. Control of perennated bermudagrass (Cynodon dactylon) and johnsongrass (Sorghum halepense) in sugarcane (Saccharum spp. hybrids). Weed Technol., v. 12, p.128-133, 1998.

ROLIM, J.C.; CHRISTOFFOLETI, P.J. Tolerância de cultivares de cana-de-açúcar (Saccharum spp) ao herbicida tebuthiuron. Campinas. CONGRESSO BRASILEIRO DE HERBICIDAS E ERVAS DANINHAS (SBHED), 14; CONGRESO DE LA ASSOCIACION LATINO AMERICANA DE MALEZAS (ALAM), 6, 1982, Campinas. Resumos... Campinas: 1982. p.90-91. 
SHAW, D.R.; WIXSON, M.B. Postemergence combinations of imazaquin or imazethapyr with AC 263,222 for weed control in soybean (Glycine max). Weed Sci., v.39, p.644-649, 1991.

WIXSON, M.B.; SHAW, D.R. Effects of adjuvants on weed control and soybean (Glycine max) tolerance with AC 263,222. Weed Technol., v.5, p.817-822, 1991a.
WIXSON, M.B.; SHAW, D.R. Use of AC 263,222 for sicklepod (Cassia obtusifolia) control in soybean (Glycine max). Weed Technol., v.5, p. 434-438, $1991 b$.

WIXSON, M.B.; SHAW, D.R. Effects of soil-applied AC 263,222 on crops rotated with soybean (Glycine max). Weed Technol., v.6, p.276-279, 1992. 